\title{
Implementación de la Ley 618 “Ley de Higiene y Seguridad Laboral" en las empresas de zonas francas del sector tabaco de la ciudad de Estelí 2015 ${ }^{1}$
}

\author{
Axel Joel Falcón Matute ${ }^{2}$ \\ Rene Alberto Rodríguez Solórzano ${ }^{3}$ \\ Jagdiel Mauricio Manzanares García ${ }^{4}$ \\ Beverly Castillo Herrera ${ }^{5}$
}

\section{RESUMEN}

El presente trabajo está dirigido al análisis de la implementación de la Ley 618 "Ley de higiene y seguridad laboral" en las tabacaleras bajo el régimen de zonas francas en el departamento de Estelí. Según el nivel de conocimiento de esta investigación es aplicada debido a que se analiza el cumplimiento de la ley 618, según el enfoque filosófico la presente investigación se considera de tipo cuantitativa. El universo de estudio está constituido por las 15 empresas de zonas francas del sector tabaco. La técnica de recolección de datos son la encuesta y la entrevista dirigida a los responsables de higiene y seguridad de las empresas y a los responsables de las instituciones del estado INSS y MITRAB. En los principales resultados se describe la organización que las empresas de zonas francas del sector tabaco tienen en cuanto a higiene y seguridad se trata.

Palabras clave: Higiene, Seguridad, Zona Franca, Tabaco.

Recibido: 10 de diciembre de 2015

Aceptado: 4 de marzo de 2016

1 Este artículo se basa en la investigación titulad: Implementación de la Ley 618 "Ley de Higiene y Seguridad Laboral" en las empresas de zonas francas del sector tabaco de la ciudad de Estelí 2015. Para optar al título de Licenciatura en Administración de empresas en la UNAN-Managua, FAREM-Estelí.

2 UNAN-Managua, FAREM-Estelí. Correo electrónico axel.falcon@yahoo.com

3 UNAN-Managua, FAREM-Estelí. Correo electrónico rene.rodsol@gmail.com

4 UNAN-Managua, FAREM-Estelí. Correo electrónico jahdieljm10@gmail.com

5 Candidata a doctora en Ciencias Sociales por la Universidad del Zulia, Venezuela. Maestría en Ciencias Sociales por la Universidad de Guadalajara. Docente titular de la UNAN-Managua, FAREM-Estelí. Correo electrónico: beverly. castillo@yahoo.com 


\title{
Implementation of the Law 618 "Occupational Health and Safety" at the enterprises of Tobacco in the city of Estelí 2015
}

\begin{abstract}
This research paper is done to the analysis of the implementation of the Law 618 "Law of Occupational Health and Safety" at the tobacco factories in Estelí. This is an applied research due to the analysis of the fulfilment of the 618 , also is considered as quantitative. The universe of study is 15 enterprises of Tobacco. The data was collected using two different approaches: the survey and the interview done to responsible of the area of hygiene and safety at the tobacco factories and to the responsible of the INSS and MITRAB. In the main results, the organization of the area of Occupational Health and Safety at the tobacco factories are described.
\end{abstract}

Key words: Hygiene, safety, tobacco 


\section{INTRODUCCIÓN}

El presente estudio describe la implementación de las normativas de la ley 618 (Ley de higiene y seguridad laboral) en las zonas francas del sector tabaco de la ciudad de Estelí, los accidentes laborales aumentan en estas empresas cuando no se ponen en práctica normativas de la ley, ocasionando un daño a futuro en la salud y seguridad de los trabajadores.

Uno de los principales problemas que se presenta en los trabajadores, proviene de la manipulación del tabaco durante la elaboración del puro, que representa riesgo para la salud y seguridad, porque la materia prima que se utiliza, contiene químicos nocivos para el ser humano.

Las condiciones laborales son un factores de riesgo que propicia al peligro y riesgo a los trabajadores causando accidentes laborales que percuten en los trabajadores y los empleadores de las empresas los cuales están propensos a pagar multas en dependencia del tipo de accidente que ocurra.

La ley 618 de higiene y seguridad laboral, tiene por objeto establecer el conjunto de disposiciones mínimas que, en materia de higiene y seguridad del trabajo, el estado, los empleadores y los trabajadores deberán promover y utilizar en los centros de trabajo, mediante la promoción, intervención, vigilancia y establecimiento de acciones para proteger a los trabajadores en el desempeño de sus labores.

Las empresas que ven la inversión en la higiene y seguridad de los trabajadores como un gasto podrían exponerse a un mayor riesgo de accidentes laborales. La compra de equipo de protección personal, mejora de infraestructuras y las capacitaciones dentro de la empresa podrían disminuir las utilidades y afectar su funcionamiento económico.
Como parte de las medidas, podrían implementarse capacitaciones donde los empleadores luego de invertir en equipo de protección personal para cada uso. Exigirían medidas drásticas y permanentes de adaptación para prevenir riesgo y enfermedades en los trabajadores. Se pretende que al aplicar la ley 618 de Higiene y seguridad laboral en las zonas francas no sea visto como un gasto, sino como una inversión en la salvaguardia de los trabajadores que le pueda evitar problemas, afectándole el ambiente de trabajo y la imagen de la empresa.

Esta investigación será de utilidad tanto como para los empleadores como para los trabajadores, el Ministerio del Trabajo (MITRAB), e Instituto de Seguridad Social (INSS) ya que aportara aspectos teóricos y prácticos de la aplicación de la ley que servirá para regular las normativas de higiene y seguridad de las empresas, exigiéndole el cumplimiento de la misma, y las supervisiones periódicas por parte de un supervisor designado que se encargue de conocer las necesidades de los empleados y darle respuesta a los mismos.

Desde el punto de vista académico servirá como documento de estudio ya que formara parte del material bibliográfico, el cual servirá tanto a docentes y futuros profesionales que requieran de información acerca de la ley 618 de Higiene y Seguridad implementada en las zonas francas.

\section{Conceptos Básicos.}

\section{Concepto de Empresa.}

La empresa es una organización social que realiza un conjunto de actividades y utiliza una gran variedad de recursos (financieros, materiales, tecnológicos y humanos) para lograr determinados objetivos, como la satisfacción de una necesidad o deseo de su mercado meta con la finalidad de lucrar o no; y que es construida a partir de conversaciones específicas basadas en compromisos mutuos entre las personas que la conforman. (Ivan, 2007). 


\section{Concepto de Zona Franca ${ }^{1}$}

Toda área del territorio nacional, debidamente cercada, sin población residente, bajo la vigilancia de la Dirección General de Aduanas, sometido a control aduanero especial, con el único propósito de albergar empresas que se dediquen a la importación de materias primas, transformarlas en productos terminados $\mathrm{y}$ venderlos en el exterior. También estas empresas pueden comprar sus materias primas en el país, procesarlas y exportar el producto terminado. Las Zonas Francas, deben considerarse como situadas fuera del territorio nacional para efectos fiscales. Las Zonas podrán estar ubicadas en cualquier parte del territorio nacional, excepto en áreas protegidas y parques nacionales.

\section{Concepto de Higiene ${ }^{2}$}

Conjunto de normas y procedimientos tendientes a la protección de la integridad física y mental del trabajador, preservándolo de los riesgos de salud inherentes a las tareas del cargo y al ambiente físico donde se ejecutan.

Está relacionada con el diagnóstico y la prevención de enfermedades ocupacionales a partir del estudio y control de dos variables: el hombre - y su ambiente de trabajo, es decir que posee un carácter eminentemente preventivo, ya que se dirige a la salud y a la comodidad del empleado, evitando que éste enferme o se ausente de manera provisional o definitiva del trabajo.

Conforma un conjunto de conocimientos y técnicas dedicados a reconocer, evaluar y controlar aquellos factores del ambiente, psicológicos o tensionales, que provienen, del trabajo y pueden causar enfermedades o deteriorar la salud.

1 Comisión Nacional de Zonas Francas (2014). Conceptos Generales. Consultado el 24 de abril del 2013. Disponible en: /http://www.cnzf.gob.ni/?q=es/quienes-somos/conceptosgenerales

2 CIMO (2002) Gestio Polis Consultado13 Julio.2015/http:// www.gestiopolis.com/organizacion-talento/seguridad-e-higieneen-el-trabajo.htm

\section{Concepto de Seguridad ${ }^{3}$}

Conjunto de medidas técnicas, educacionales, médicas y psicológicas empleados para prevenir accidentes, tendientes a eliminar las condiciones inseguras del ambiente y a instruir o convencer a las personas acerca de la necesidad de implementación de prácticas preventivas.

Según el esquema de organización de la empresa, los servicios de seguridad tienen el objetivo de establecer normas y procedimientos, poniendo en práctica los recursos posibles para conseguir la prevención de accidentes y controlando los resultados obtenidos. El programa debe ser establecido mediante la aplicación de medidas de seguridad adecuadas, llevadas a cabo por medio del trabajo en equipo.

La seguridad es responsabilidad de Línea y una función de staff. Cada supervisor es responsable de los asuntos de seguridad de su área, aunque exista en la organización un organismo de seguridad para asesorar a todas las áreas.

\section{MATERIALES Y MÉTODOS}

El universo de estudio lo constituyen 15 constituidas como zonas francas del sector tabaco ubicadas en la ciudad de Estelí que son: 1) NACSA; 2) Puros de Estelí E.A.; 3) Scandinavian Tobacco Group, S.A.; 4) Drew State Tobaco, CO; 5) Plasencia Cigars, S.A.; 6) Tabacalera Tambor de Estelí; 7) Perdomo, S.A.; 8) Tabolisa; 9) My fathers cigars; 10) Tavicusa; 11) Joya de Nicaragua, S.A.; 12) Tacuba; 13) A.J. Fernández, S.A.; y 14) Nica Tabaco

Con un universo de 15 empresas de zonas francas, un margen de error del 5\% y con un nivel de confianza del 95\% el tamaño de la muestra es de 14.46 es decir 14 empresas de zonas francas de la ciudad de Estelí.

3 CIMO (2002) Gestio Polis Consultado13 Julio.2015/http:// www.gestiopolis.com/organizacion-talento/seguridad-e-higiene-en-el-trabajo.htm. 
Según el tipo de investigación el muestreo es Probabilístico, la selección de la muestra se hizo en forma aleatoria simple, donde cualquiera de las empresas de zonas francas del universo de estudio tiene la misma probabilidad de ser seleccionada.

Se utilizaron dos técnicas de recolección de datos en las 14 empresas del sector tabaco: la encuesta y la entrevista. La encuesta fue dirigida a los empleadores y empleados de las empresas del sector tabaco y la entrevista a las instituciones del estado (INSS-MITRAB). Además, se realizó investigación documental, revisando libros, tesis, y páginas web.

Para analizar la información estadística se elaboraron tablas de frecuencia. El informe final se trabajó en base a una guía facilitada por la universidad.

\section{RESULTADOS Y DISCUSIÓN}

Las 15 empresas encuestadas del sector tabaco cuentan con comisión mixta, dado que es uno de los requisitos indispensables para los trámites de licencia en materia de higiene y seguridad para y puedan operar como zona franca.

E1 $93 \%$ afirma tener un plan anual de comisión mixta y el $7 \%$ manifiesta que todavía no cuentan con un plan. Sólo una empresa expreso tener su plan anual de Comisión Mixta, y afirmaron que se encuentran realizando esta obligación.

\section{Cuadro No 1. Valoración de la seguridad de los trabajadores}

Universo: 14 Empresas Tabacaleras

\begin{tabular}{lrrr}
\hline \multicolumn{1}{c}{ Datos } & Cantidad & \% \\
\hline Buena & 9 & 64 \\
Regular & 4 & 29 \\
Mala & 1 & 7 \\
TOTAL & 14 & 100 \\
\hline
\end{tabular}

Fuente: Datos primarios de la encuesta, Mayo, 2015
Al preguntar sobre la seguridad de los trabajadores dentro de las empresas, el 64\% afirma que su seguridad brindada es buena, el $29 \%$ brindan una seguridad regular, y el $7 \%$ es una seguridad mala, podemos observar que no existe conciencia en su totalidad en cuanto en materia de seguridad para los trabajadores. En la cual se puede notar que falta hacer mejoras en las empresas para garantizar una mejor seguridad.

\section{Cuadro No 2. Frecuencia de las capacitaciones} Universo: 14 Empresas

\begin{tabular}{lrrr}
\hline \multicolumn{1}{c}{ Frecuencia } & Cantidad & \% \\
\hline Semestral & 5 & 36 \\
Trimestral & 7 & 50 \\
Mensual & 2 & 14 \\
Otras & - & - \\
TOTAL & 14 & 100 \\
\hline
\end{tabular}

Fuente: Datos primarios de la encuesta, Mayo, 2015

Cuando se les pregunto con qué frecuencia ellos realizaban capacitaciones a sus trabajadores, el $36 \%$ las realizan semestralmente, el 50\% realizan capacitaciones trimestrales, y un 14\% respondió que realizan capacitaciones cada mes, se puede decir que más de la mitad de las empresas tienen el interés que sus trabajadores sean capacitados con mayor frecuencia.

\section{Cuadro No 3. Equipo de protección personal (EPP) que disponen los trabajadores}

Universo: 14 Empresas

\begin{tabular}{lcr}
\hline Equipos de protección & Cantidad & \% \\
\hline Cascos & 7 & 50 \\
Guantes & 8 & 57 \\
Mascarillas & 9 & 64 \\
orejeras & 6 & 43 \\
Gabacha / Chalecos & 8 & 57 \\
Cinturón & 7 & 50 \\
Arnés & 1 & 7 \\
Botas & 7 & 50 \\
todas & 2 & 14 \\
\hline
\end{tabular}

Fuente: Datos primarios de la encuesta, Mayo, 2015 
El cuadro numero 3 muestra el equipo de protección personal que la mayoría de las empresas cuentan con estos equipos sobresalen con un 64\% y un $57 \%$ gabachas y guantes la materia prima y las condiciones en que laboran. Solo el $19 \%$ hace uso de los arneses por que no todas las empresas cuentan con racks para almacenar sus materiales.

\section{Cuadro No 4. Equipos que no son usados frecuentemente por personal Universo: 14 Empresas.}

\begin{tabular}{lrr}
\hline Equipos de protección & Cantidad & \% \\
\hline Cascos & 1 & 7 \\
Guantes & 2 & 14 \\
Mascarillas & 6 & 42 \\
Orejeras & 3 & 21 \\
Gabacha / Chalecos & 3 & 21 \\
Cinturón & 11 & 79 \\
Arnés & 1 & 7 \\
Botas & 4 & 29 \\
Todas & 1 & 7 \\
\hline
\end{tabular}

Fuente: Datos primarios de la encuesta, Mayo, 2015

Al preguntar sobre el equipo de protección personal que los trabajadores no usan con frecuencia esta el $7 \%$ el casco, $14 \%$ los guantes, $42 \%$ mascarillas, $21 \%$ orejeras, de igual manera con el 21\% gabachas/chalecos, 79\% los cinturones, el 7\% los arnés, y el 29\% las botas. Con relación a los motivos que intervienen a que los trabajadores no usen el equipo de protección personal el 50\% afirma, ya que les provoca incomodidad para que realizar sus labores, y se acostumbren a utilizarlos.

\section{Cuadro No 5. Medidas utilizadas para controlar el uso de EPP}

Universo: 14 Empresas Tabacaleras

\begin{tabular}{lrr}
\hline \multicolumn{1}{c}{ Medidas } & Cantidad & \% \\
\hline Supervisión & 11 & 79 \\
Memorándum & 3 & 21 \\
Concientizar & 4 & 29 \\
Otras & 8 & 57 \\
\hline
\end{tabular}

Fuente: Datos primarios de la encuesta, Mayo, 2015
En relación al equipo de protección se les pregunto las medidas que utilizan para controlar que los trabajadores utilicen el equipo de protección y el 79\% realizan supervisión, el 21\% utilizan memorándum cuando el trabajador no acata órdenes y el $29 \%$ concientiza a sus trabajadores los equipos para evitar accidentes o enfermedades que les afecte en algún momento.

\section{Cuadro No 6. Frecuencia con la que realizan exámenes médicos}

Universo: 14 Empresas Tabacaleras

\begin{tabular}{lrr}
\hline \multicolumn{1}{r}{ Frecuencia } & Cantidad & \% \\
\hline Trimestral & 0 & 0 \\
Semestral & 1 & 7 \\
Anual & 13 & 93 \\
Total & 14 & 100 \\
\hline
\end{tabular}

Fuente: Datos primarios de la encuesta, Mayo, 2015

Con respecto a los exámenes médicos el 93\% los realiza anualmente, lo consideran por año es un buen tiempo para hacer estos exámenes, y la ley 618 no estipula la frecuencia deben realizarse los exámenes médicos, por tanto ellos no incumplen con la ley.

\section{Rol del MITRAB en la aplicación de la ley 618}

El Ministerio del Trabajo a medida que han crecido las empresas de zonas francas del sector tabaco en la ciudad de Estelí, ha venido realizando un arduo trabajo en coordinación con la Asociación de Tabacaleros conformada principalmente por los gerentes de las empresas del sector.

Al inicio de cada año se establecen las coordinaciones del plan anual con los representantes de insectoría del INSS, y se definen las fechas y actividades a realizar en el año, para realizar una recapitulación de las principales incidencias ocurridas durante los años anteriores.

El MITRAB como institución que aplica la ley 618 se acciona según el acta de inspección, por ello se revisa primordialmente lo que establece la ley no se guían 
por tamaño ni características individuales, sino que se trata de manera en general, a las empresas de tabaco que trabajan con mayor cantidad de persona tienen mayor riesgo que ocurra un accidente de trabajo, por esto que se les exige un poco más y siempre regulado a lo establecido en la ley 618 .

Las empresas están adoptando prácticas positivas para cumplir con las medidas necesarias para garantizar el cumplimiento de la ley a través de la contratación de responsables de higiene y seguridad y cumplir con las normativas establecidas por la ley 618. Otras medidas positivas implementada en las empresas es garantizar la satisfacción de los trabajadores en las medidas y las condiciones de trabajado que las empresas les brindan.

\section{Rol del INSS}

El Instituto Nacional de Seguridad Social (INSS) está trabajando en conjunto con la asociación de tabacaleros a manera de buscar los mejores beneficios en cuanto a higiene y seguridad para los trabajadores de las empresas del sector tabaco de la ciudad de Estelí.

Cuando se realizan inspecciones a las empresas el INSS espera garantizar el cumplimiento de las normas y que cumplan las medidas necesarias para la seguridad de los trabajadores. El INSS pretende que las empresas tengan una cultura por parte de los empleadores para cumplir con la dotación y uso adecuado de los equipos de protección.

El INSS hace uso de la ley 618 para orientar al empleador que debe de cumplir con lo establecido en las normativas de la ley sin embargo el INSS es el encargado de garantizar la aplicación de la ley de seguro social ley 974.

Al igual que el MITRAB el INSS sugiere a las empresas que se realicen capacitaciones sus trabajadores en materia de higiene y seguridad, de esta manera se garantiza que exista menos accidentes laborales y enfermedades que conlleven al deterioro de la salud del trabajador.

\section{Cumplimiento de la ley 618 en las empresas}

Según los datos reflejados, las empresas tienden a recibir primordialmente inspecciones por parte del INSS, con un 93\% siendo el INSS es el encargado de garantizar la seguridad e higiene de los trabajadores en el campo laboral mientras que en segunda instancia el MITRAB con un 79\%. Garantiza que se de el cumplimiento de las normativas de la ley 618.

\section{Cuadro No 7. Requisitos con los que cumple la empresa}

\section{Universo: 14 Empresas Tabacaleras}

\begin{tabular}{lrc}
\hline \multicolumn{1}{c}{ Frecuencia } & Cantidad & $\mathbf{\%}$ \\
\hline Comisión mixta & 14 & 100 \\
Plan anual de comisión mixta & 14 & 100 \\
Reglamento interno de funcionamiento & 14 & 100 \\
Reglamento técnico organizativo & 13 & 93 \\
Plan de emergencia & 12 & 86 \\
Brigada contra incendios & 12 & 86 \\
Exámenes ocupacionales & 14 & 100 \\
Señalización & 14 & 100 \\
\hline
\end{tabular}

Fuente: Datos primarios de la encuesta, Mayo, 2015

Las empresas en su mayoría cumplen con los requisitos exigidos las instituciones del estado y contemplados en la ley, de igual manera se encuentra un déficit en el cumplimiento de las brigadas contraincendios como se ve reflejado en la tabla con un $83 \%$, al no cumplir con este requisito las empresas no podrán obtener la aprobación del plan de emergencia de igual manera la licencia de higiene y seguridad.

En su a gran mayoría de las empresas reciben seminarios por parte de las instituciones del estado solamente con los representantes de la comisión mixta de la empresa y el personal del área de Recursos Humanos una vez al año. Lo cual esta represento por un 43\%. Lo cual indica que las instituciones del estado tienen poco interés en capacitar y brindar charlas al personal que labora en las empresas de zona franca. 
Según a las empresas a las que se les realizo encuesta, muestran como resultado que las instituciones les prestan poco interés en cuanto a las inspecciones ya que estas solo se realizan de 1 a 2 veces en el año, tomando como referencia un porcentaje de $58 \%$ semestral y el $21 \%$ anual.

Según las empresas deben de cumplir con todos los requisitos exigidos por la ley 618 en cuanto a documentación. Las cuales se encuentran reflejado en la siguiente tabla todas las empresas encuestadas están trabajando para cumplir lo establecido en la ley en un $100 \%$ siendo conscientes de los requisitos que deben cumplir.

\section{Descripciones de multas o sanciones}

El 79\% de las Tabacaleras no han sido multadas por la ley 618 , mientras un $21 \%$ han sido multadas por el incumplimiento de la Ley 974 "Ley de Seguridad Social".

Según la clasificación de las multas las empresas que han sido multadas 2 de ellas representado con un $67 \%$ en clasificación de multa leve debido a que la falta no fue no ameritaba otro tipo de sanción además que era la primera vez que esta empresa presentaba problemas. Por lo contrario una empresa fue multada con una clasificación grave ya que era reincidencia en la falta al por no reportar el accidente ocurrido.

Dos empresas que recibieron multa por parte del INSS. Fueron por reportar mal un accidente y la causa fue al llenar mal la hoja NAT representado con un 67\%, y con un $33 \%$ por no reportar un accidente, al verse en este tipo de situación la empresa tiene que asumir todo los gasto del trabajador. Cabe destacar que solo una empresa ha sido multada por parte del MITRAB con la ley 618 debido a que el trabajador afectado se quejó debido a que la empresa se negó a llenarle la hoja NAT.

La sugerencia que más porcentaje tiene es que exista mayor comunicación entre las empresas y las instituciones del estado con un 57\%. Al haber mayor comunicación los responsables de Higiene y Seguridad sabrán como desempeñar mejor su rol y eficiencia para las buenas prácticas de Higiene y Seguridad en la empresa.

El 14\% considera otras sugerencias como: cumplir con los requisitos para adquirir la licencia y poder operar ya que las empresas al tener la licencia de higiene y seguridad minimiza los riesgos de peligro para los trabajadores.

\section{Propuesta de Estrategias de la empresa.(FODA)}

De acuerdo a la información recopilada tanto en las encuesta y las entrevista a los encargados de la higiene y seguridad de las empresas tabacaleras bajo el régimen de zona franca. Como también a los encargados de las instituciones del estado INSS y MITRAB se identificaron: las fortalezas, oportunidades, debilidades y amenazas de las zonas francas. 


\begin{tabular}{|c|c|}
\hline FORTALEZAS & OPORTUNIDADES \\
\hline 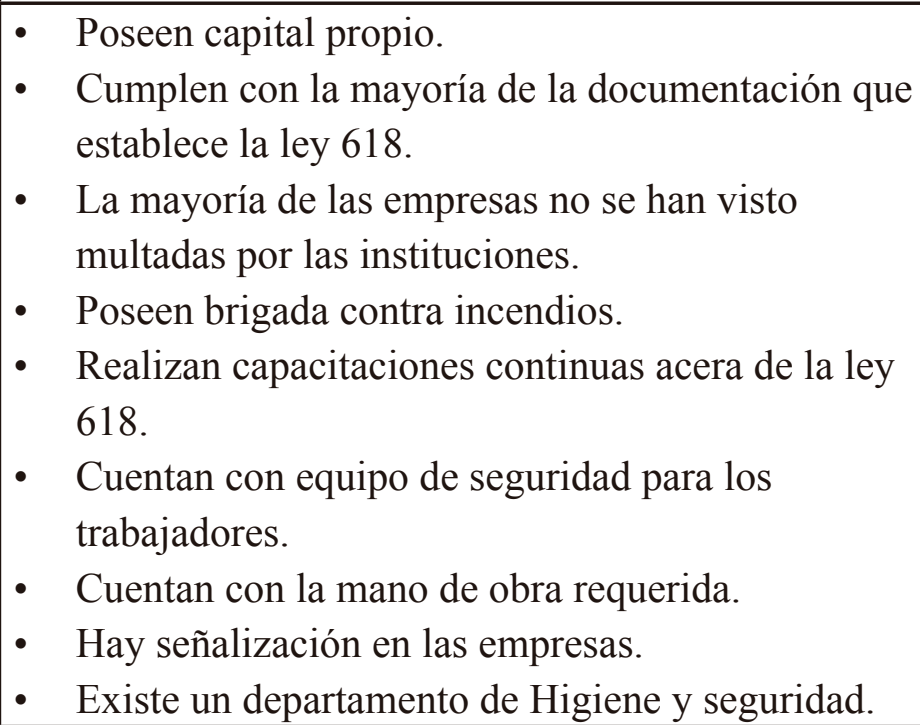 & $\begin{array}{l}\text { - Se encuentran bajo el régimen de zonas francas. } \\
\text { - Trabajan en conjunto perteneciendo a las } \\
\text { asociaciones de tabacaleros. } \\
\text { - Incrementación de la demanda y generación de } \\
\text { empleo. } \\
\text { - Desarrollo económico en la cuidad. } \\
\text { - Integran a la comisión mixta trabajadores de la } \\
\text { - } \quad \text { Trabajan en conjunto con las instituciones del } \\
\text { estado encargadas por velar por la seguridad de } \\
\text { los trabajadores. }\end{array}$ \\
\hline DEBILIDADES & AMENAZAS \\
\hline $\begin{array}{l}\text { - Poca inversión en la seguridad e higiene de los } \\
\text { trabajadores. } \\
\text { Poca rigurosidad en la exigencia de equipo de } \\
\text { protección personal a los trabajadores. } \\
\text { - No cuentan con una licencia de higiene y } \\
\text { seguridad industrial. }\end{array}$ & $\begin{array}{l}\text { - } \text { Incrementación en las multas. } \\
\text { - Cierre temporal o parcial de la empresa. } \\
\text { Pocas visitas por parte de las instituciones del } \\
\text { estado provocando irregularidades en la eficiencia } \\
\text { de la seguridad. }\end{array}$ \\
\hline
\end{tabular}

Propuesta de Estrategias de los trabajadores. (FODA)

\begin{tabular}{|c|c|}
\hline FORTALEZAS & OPORTUNIDADES \\
\hline $\begin{array}{l}\text { - Poseen los equipo de protección personal } \\
\text { necesarios. } \\
\text { - Cuentan con comisión mixta. } \\
\text { - Cuenta con los equipos de seguridad. }\end{array}$ & $\begin{array}{l}\text { - Poseen condiciones de trabajos seguros. } \\
\text { - Integración a las brigadas existente en las } \\
\text { empresas. } \\
\text { - Adquisición de nuevo equipos de protección } \\
\text { necesarios. } \\
\text { - Cuentan con convenio colectivos. }\end{array}$ \\
\hline DEBILIDADES & AMENAZAS \\
\hline 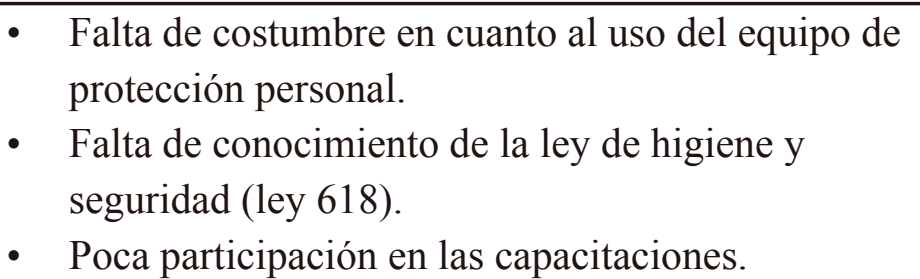 & $\begin{array}{l}\text { - } \quad \text { El poco interés de las instituciones del estado. } \\
\text { - Posibilidad de adquirir una enfermedad } \\
\text { profesional. } \\
\text { - Exposición a riesgo por las herramientas } \\
\text { utilizadas. }\end{array}$ \\
\hline
\end{tabular}




\section{Propuesta MECA}

\section{Maximizar las Fortalezas}

- Continuar poniendo en práctica los instrumentos para cumplir lo establecido en la ley 618.

- Conservar el interés en la sostenibilidad de empleo y la inversión en el capital humano logrando de esta manera el mejor recurso del producto terminado.

- Promover la higiene y seguridad en las distintas áreas de la empresa reduciendo la incidencia de accidentes laborales.

\section{Explotar las Oportunidades}

- Aumentar la capacidad de trabajadores logrando de esta manera mayor productividad y efectividad del trabajo.

- Promover la comunicación entre los representantes de las instituciones del estado, asociación de tabacaleros y comisión mixta de las empresas para conocer las problemáticas y debilidades que se presenten brindando solución en tiempo y forma.

- Fomentar estrategias para maximizar el desarrollo económico en la ciudad, cumpliendo de esta manera con la responsabilidad social empresarial.

\section{Corregir las Debilidades}

- Realizar capacitaciones en cuanto a higiene y seguridad de los trabajadores para la prevención de accidentes laborales.

- Aumentar la rigurosidad y atención a las áreas de mayor riesgo y peligro para la vida del trabajador.

- Mayor exigencia por parte de los responsables de higiene y seguridad sobre el uso del equipo de protección personal.

\section{Afrontar la amenaza}

Realizar la certificación de la licencia de higiene y seguridad industrial en las empresas de zonas francas del sector tabaco, salvaguardando las operaciones de la misma cumpliendo con las orientaciones brindadas por las instituciones del estado.
Estrategia para mejora la implementación de la ley 618 por parte de las empresas tabacaleras bajo el régimen de zonas francas.

Estrategia 1: Desarrollar la inversión en la seguridad de los trabajadores.

Objetivo: Mejorar las condiciones en las que laboran los trabajadores de las empresas tabacaleras bajo el régimen de zona franca.

\section{Actividades:}

- Crear una cuenta destinada a los gastos en cuanto a materiales de higiene y seguridad.

- Detallar cantidad necesaria del equipo de protección.

- Cotizar equipo de protección personal.

- Comprar equipo de protección personal para los trabajadores.

- Capacitación para el uso adecuado del equipo de protección.

Estrategia 2: Concientizar a los Trabajadores.

Objetivo: Crear una cultura de higiene y seguridad en los trabajadores de las empresas de zona Franca.

\section{Actividades:}

- Concientizar al personal sobre el uso de equipo de protección personal.

- Capacitar a los trabajadores en cuanto al uso del equipo de protección personal.

- Monitoreo por parte de la comisión mixta en el uso del equipo de protección personal.

Estrategia 3: Mejoramiento en la infra estructura y las condiciones de trabajo.

Objetivo: Garantizar el buen ambiente laboral a los trabajadores, dentro de las instalaciones de la empresa. 


\section{Actividades:}

- Evaluación inicial de riesgos.

- Análisis de la evaluación de riesgo.

- Decidir sobre las posibles soluciones.

- Ejecutar las soluciones.

Estrategia 4: Aplicar lo establecido en el reglamento interno de las empresas por las falta de uso del equipo de protección personal.

Objetivo: Determinar un sistema de control rutinario sobre el uso de equipo de protección personal.

\section{Actividades:}

- Realizar supervisiones en todas las áreas de la empresa.

- Evaluar las principales áreas de la empresa donde no se hace uso del equipo de protección personal.

- Concientizar al personal en cuanto al uso de protección personal.

- Suspensión temporal de trabajo a los trabajadores de la empresa que no hace uso del equipo de protección personal.

\section{CONCLUSIONES}

Las empresas al incumplir con las reglas y normas estipuladas en la Ley 618, se hace acreedora a recibir una multa por parte de las instituciones del estado siendo sancionadas para que en futuras situaciones o circunstancias similares, de menor o mayor magnitud velen por la seguridad y el bienestar de todos y cada uno de los trabajadores, y no únicamente por los intereses personales y lucrativos de cada empresa.

Se puede mencionar que estas empresas en su mayoría no implementan la ley de higiene y seguridad, ya que existe desinterés por parte de los empleadores en invertir para el bienestar de sus trabajadores, al realizar el trabajo de campo se encontraron 3 empresas que no cuentan con un responsable de higiene y seguridad lo que conlleva a que estas empresas presenten mayores riesgo a las seguridad e higiene de los trabajadores.
La participación activa de los trabajadores en la labor continua de prevención de accidentes es un factor clave para el éxito de cualquier sistema de prevención de accidentes. La creación de un ambiente seguro en el trabajo implica cumplir con las normas y procedimientos, sin pasar por alto ninguno de los factores que intervienen en la conformación de la seguridad del trabajador.

La mayoría de los accidentes de trabajo en las empresas del tabaco ocurren por falta de atención e interés por parte del empleador y de los trabajadores, al no utilizar el equipo de protección personal adecuado además aducen que los trabajadores muestran renuencia al uso del equipo, ya que estos los incomodan al realizar sus labores.

Los trabajadores en su mayoría aducen ser conscientes de estar prop ensos a adquirir enfermedades producto de la manipulación del tabaco al igual, el no hacer uso del equipo de protección durante sus jornadas de trabajo.

Las instituciones del estado encargada por el cumplimiento y aplicación de la ley están trabajando arduamente con las empresas de zona franca del sector tabaco realizando inspecciones continuas a estas, con el objetivo de garantizar que los trabajadores se encuentren en un ambiente fuera de peligro.

Con los resultados obtenidos en las encuestas realizadas en las empresas de zonas francas de la ciudad de Estelí al igual que con las entrevistas a las instituciones del estado (INNS-MITRAB), hacemos referencia que se cumple con la hipótesis del trabajo investigativo debido a que no existen empresas que se han visto multadas debido al incumplimiento de la ley 618 y los llamados de atención al momento que se ejecutan las inspecciones por parte de las instituciones del estado.

No todas las empresas evaluadas cumplen con los principales parámetros exigidos por los reglamentos de higiene y seguridad que establecen la ley y las 
instituciones del estado para mantener un ambiente de trabajo seguro e higiénico sin embargo el MITRAB es algo flexible al momento de realizar las inspecciones.

\section{BIBLIOGRAFÍA}

Ley 618 Asamblea Nacional. Ministerio del Trabajo. Obtenido de Ministerio del Trabajo: http://www. mitrab.gob.ni/documentos/leyes/Ley618Nic.pdf/ view fecha de consulta (15 de Noviembre)

Acuña Martina, L. I. (2009). Practicas ynormas sobre el uso de procteccion entre los/as obreros/as de la fabrica de tabaco de productos termenados. Esteli. Fecha de consulta 24 abril 2015.

Comision nacional de zonas francas (2014). CNZF. Obtenido de CNZF: http://www.cnzf.gob.ni/?q=es/ quienes-somos/conceptos-generales. Fecha de consulta 24 de abril 2015. 